males and 6 females; mean age 10.8 years) and 57 developed other symptoms: a) during or immediately after, or b) at least 30 minutes after viewing the TV program. Older children tended to develop symptoms, including seizures, more frequently than younger children. Symptoms in Group a) included headache, nausea, vomiting, blurred vision or vertigo ( 28 children [2.6\%]), and delayed-onset symptoms in Group b) were similar or minor, resembling motion sickness (29[2.7\% of viewers]). Individual or family history of seizures was obtained in $80 \%$ patients with seizures, compared to only $29 \%$ of patients with other symptoms and $27 \%$ with minor delayed-onset symptoms $(\mathrm{p}<0.001)$. Compared with nonaffected children, significantly more affected children had concentrated on watching the TV program, watched it at a short distance from the screen, and in a dimly lit room. (Furusho J, Suzuki M, Tazaki I et al. A comparison survey of seizures and other symptoms of Pokemon phenomenon. Pediatr Neurol Nov 2002;27:350-355). (Respond: Dr Furusho,Department of Pediatrics, Showa University School of Medicine, 1-5-8 Hatanodai Shinagawaku, Tokyo, 142-8666, Japan).

COMMENT. Pokemon phenomenon was a significant social problem in Japan and resulted in many studies regarding the adverse effects of cathode ray tube images and TV viewing, conditions facilitating induction of photosensitive seizures, and other symptoms. Children with seizures had a heightened predisposition, and viewing conditions had not been optimal. Guidelines for TV viewers cautioned against sitting close to the screen and in a dark room. Blue sunglasses were recommended for viewers susceptible to photosensitive epilepsy. Symptoms occurring other than seizures included headache and dizziness, resembling motion sickness. See Progress in Pediatric Neurology III, PNB Publishers, 1997;pp 64-67, for articles on visually induced seizures, including video-game epilepsy, self-induced photogenic epilepsy, and reading epilepsy.

\title{
ANTIEPILEPTIC DRUG-INDUCED FETAL MALFORMATIONS
}

Women with epilepsy attending a single maternity clinic, Helsinki University Central Hospital, Finland, were followed prospectively during 970 pregnancies in 1980 through 1998. Of the 979 offspring, 740 were exposed to maternal antiepileptic drugs (AED) during the first trimester of pregnancy and 239 were not exposed. Major malformations occurred in 28 fetuses $(3.8 \%)$ exposed to AED and in $2(0.8 \%)$ not exposed $(p=0.02)$. The occurrence of major malformations was independently associated with the use of carbamazepine $(\mathrm{p}=0.05)$, valproate $(\mathrm{p}=0.003)$, oxycarbamazepine $(\mathrm{p}=0.04)$, low serum folate concentration $(<4.4 \mathrm{nmol} / \mathrm{L})$, and low maternal level of education. AED levels and serum folate were obtained at the end of the first trimester. No correlation was observed between dose or serum concentration of AED and malformations. The risk of malformations increased as the number of AED used increased. Grand mal seizures during the first trimester were not a factor in the cause of major fetal malformations. Alcohol intake, smoking, and exposure to phenytoin, clonazepam, phenobarbital, or primidone showed no significant association with fetal malformations. (Kaaja E, Kaaja R, Hiilesmaa V. Major malformations in offspring of women with epilepsy. Neurology February (2 of 2) 2003;60:575-579). (Reprints: Dr Vilho Hiilesmaa, Department of Obstetrics and Gynecology, Helsinki University Central Hospital, PO Box 140, 00029 HUCH, Finland). 
COMMENT. Embryopathy and major fetal malformations in children of mothers with epilepsy are associated with the use of AED during the first trimester of pregnancy and not maternal epilepsy itself. Low serum folic acid concentrations and maternal level of education are additional causative factors. Valproate and carbamazepine are especially teratogenic, whereas the association with phenytoin and phenobarbital in this study is not significant. Periconceptional folate supplements are especially recommended in women using AED, although a recent study found $0.4 \mathrm{mg}$ daily of folic acid failed to reduce the risk of fetal malformations in women using AED in early pregnancy (Hernandez-Diaz S et al. N Engl J Med 2000;343:1608-1614). The appropriate dose of folic acid needs to be determined.

\section{VASCULAR AND TRAUMATIC DISORDERS}

\section{MILD HEAD INJURY AND STROKE}

Five children, ages 1 to 6 years ( 4 female, 1 male), who suffered acute hemiparesis due to ischemic stroke and striatocapsular infarction within a few minutes to 6 hours following a mild head injury are reported from the Institute of Child Health and Great Ormond Street Hospital, London, UK. The cases were identified during an analysis of 200 cases of ischemic stroke in childhood. The head injury occurred during a minor fall. All were neurologically and developmentally normal before the injury, and all made a complete or near complete recovery. Mechanisms suggested include mechanical disruption of the arterial blood flow, trauma to the arterial intima and thrombosis, or arterial spasm secondary to trauma. One child developed chicken pox 8 days later, and a pre-eruptive varicella arteritis may have sensitized the vasculature to trauma-induced thrombosis. None had signs of other trauma to suggest non-accidental injury. (Shaffer L, Rich PM, Pohl KRE, Ganesan V. Can mild head injury cause ischaemic stroke? Arch Dis Child March 2003;88:267-269). (Respond: Dr V Ganesan, The Wolfson Centre, Mecklenburgh Square, London WC1N 2AP, UK).

COMMENT. The authors cite a previous report of 8 cases of cerebral infarction following minor head trauma in children (Kieslich M et al. J Neurol Neurosurg Psychiatry 2002;73:13-16).

Progress in Pediatric Neurology III (PNB Publishers, 1997;pp447-450) includes three different reports of minor neck trauma as a cause of vertebral artery dissection and stroke occurring during sports activities: Garg BP and colleagues report 3 cases in boys playing football and mention 16 further cases in the literature (Neurology 1993;43:25552558); Sheth RJ, Bodensteiner JB et al report a girl with cerebellar infarction following minor neck injury sustained while ice-skating (Clin Pediatr 1994;33:503-505); and Lannuzel A et al report a boy with vertebral artery dissection following a judo session (Neuropediatrics 194;25:106-108). The majority of these cases were discharged taking aspirin, but less than $50 \%$ recovered. In the report of Shaffer et al, the use of aspirin was not recorded and the majority recovered. 